\title{
Jointly Optimal Power and Signature Sequence Allocation for Fading CDMA
}

\author{
Onur Kaya Sennur Ulukus \\ Department of Electrical and Computer Engineering \\ University of Maryland, College Park, MD 20742 \\ onurkaya@eng.umd.edu_ulukus@eng.umd.edu
}

\begin{abstract}
We solve for the optimum signature sequence and power allocation policies that maximize the information theoretic ergodic sum capacity of a code division multiple access (CDMA) system subject to fading. We show that at most $N$ users may transmit at any given channel state, where $N$ is the processing gain; and those users who are transmitting should be assigned orthogonal signature sequences. We also show that the power allocation policy that maximizes the capacity together with the choice of these signature sequences is single user waterfilling over sets of channel states that are favorable to each user. That is, the capacity maximizing signalling scheme is shown to dictate that the users allocate their powers and signature sequences in such a way that they always avoid interference from each other.
\end{abstract}

\section{INTRODUCTION}

An important consideration in the design of wireless communication systems is the unavoidable presence of fading, caused by the nature of the system. To maximize the overall network capacity, one should therefore exploit the variations in the channel fade levels while allocating the available resources. Resource allocation for wireless systems can be viewed in several different contexts, including SIR-based approaches and information theoretic approaches (see [1] for some references on both). We can also group the allocation schemes according to the type of resources that are allocated. Two important resources are the transmit powers and the transmit waveforms. Power control has been studied in SIR-based and information theoretic contexts for fading and non-fading channels [1]-[4], whereas waveform optimization has been studied in these two contexts for nonfading channels only [5]-[7]. Throughout this paper the objective of resource allocation is to maximize the information theoretic ergodic (expected) sum capacity, and we consider allocating both powers and the waveforms as functions of the channel state information (CSI) in order to achieve this objective. The system of interest is assumed to be a CDMA network; thus, waveforms will simply be referred to as signature sequences.

The problem of power control in the context of capacity maximization for fading channels is first studied in [2] for a single user channel, and it is shown that the optimum power allocation policy that maximizes the ergodic channel capacity subject to an average power constraint is a waterfilling of power over the inverse of the fade levels. In this policy, more power is allocated to stronger channel states; and no power is allocated for channel states below some threshold.

Although we can define the capacity for a single user channel, when we move onto multiaccess channels, the notion of capacity

This work was supported by NSF Grants ANI 02-05330 and CCR 03-11311; and ARL/CTA Grant DAAD 19-01-2-0011. has to be replaced by a region of achievable rates [8], [9]. In such channels, it is customary to consider the maximum achievable sum of rates, i.e., the sum capacity as a figure of merit. For a multiuser scalar channel, where all users transmit with the same waveform, [3] finds the optimum power allocation policy which maximizes the ergodic sum capacity. There, it is shown that, to maximize the sum capacity, the users perform single user waterfilling over disjoint sets of channel states. That is, each user transmits only when its channel state (normalized by a factor) is greater than or equal to that of all other users. Since the channel fading is a continuous random variable, the equality of the two ratios corresponds to a zero probability event. Thus, for such a channel, at most one user transmits at a given channel state with probability 1.

For vector multiple access channels, such as CDMA, [10] proposes an asymptotically optimal single user waterfilling strategy to maximize the ergodic sum capacity in the special case of a large system with random signature sequences. The more generalized version of the power control problem with arbitrary signature sequences and arbitrary number of users and processing gain is solved in [1], where the solution is shown to be a simultaneous waterfilling of powers of users, and an iterative algorithm which performs a one-user-at-a-time single user waterfilling for each user, while the powers of all other users are fixed, is shown to converge to the optimal solution. The optimum power allocation in that case is shown to dictate more than one user to transmit simultaneously in certain regions of the channel state space, provided that the signature sequences satisfy some mild conditions.

The sum capacity of a CDMA network can also be optimized as a function of the signature sequences. When each user has an average power constraint, and there is no fading in the system, [5] shows that when the number of users is less than or equal to the processing gain, the optimal strategy is to allocate orthogonal signature sequences to all users, and when the number of users is greater than the processing gain, with all users having the same average power constraints, the optimal strategy is to allocate Welch Bound Equality (WBE) [11] sequences. Reference [6] generalizes [5] to arbitrary (unequal) average power constraints, and gives the optimal signature sequence allocation as a function of the power constraints of the users. Specifically, for the case in which the number of users is greater than the processing gain, when a user has a relatively larger power constraint then the others, it is called "oversized", and such users are allocated orthogonal signature sequences; whereas the "non-oversized" users are allocated the so-called Generalized Welch-Bound-Equality 
(GWBE) sequences.

In this paper, we attack the problem of joint power and signature sequence optimization in order to maximize the ergodic sum capacity of a fading CDMA system. Specifically, we adapt the set of signature sequences and transmit powers of all users as a function of the CSI, in order to maximize the ergodic sum capacity. At each fading state, for any given arbitrary power allocation, results of [6] can be used to allocate the optimal sequences. Among those power allocations, with signature sequences chosen optimally, we find the best power allocation strategy.

We show that the optimal strategy is still a waterfilling strategy for each user, and very strikingly, at each fading state, that strategy dictates that we allocate (at most) $N$ orthogonal signature sequences to the users with best (at most) $N$ channel states (scaled by a factor as in [3]). Moreover, the other users with worse channel states than the users with orthogonal sequences do not transmit at those particular channel states. This means that there are no users in the system which are allocated GWBE sequences and are yet transmitting with nonzero powers. Thus, in contrast to signature sequence optimization for non-fading channels, GWBE sequences are never used in transmissions; more precisely, they are used only with zero probability.

Our solution resembles [3] in the sense that there is an ordering of channel states that determines which users will transmit, but it also resembles the solution in [2] in that once we know which users will transmit at each channel state, all users will choose their powers as if they are alone in the system, i.e., they will perform single user waterfilling over favorable regions of the channel state space. This result shows that, when we have the opportunity to control both the signature sequences and the powers of the users, the users completely avoid each other, i.e., certain groups of users transmit on disjoint sets of channel states, and within each group of users that transmit at the same channel state, users place themselves orthogonal to each other in the signature sequence space, thus avoiding any possible interference.

Lastly we provide an iterative algorithm that is guaranteed to converge to the optimal power and signature sequence allocation. The algorithm performs a one-user-at-a-time waterfilling, and converges to the optimum solution described above.

\section{PRoblem DEFinition}

We consider a CDMA system with processing gain $N$, where all $K$ users transmit to a single receiver. In the presence of fading and AWGN, the received signal is given in the vector form by [12],

$$
\mathbf{r}=\sum_{i=1}^{K} \sqrt{p_{i} h_{i}} b_{i} \mathbf{s}_{i}+\mathbf{n}
$$

where $\mathbf{s}_{i}=\left[s_{i 1}, \cdots, s_{i N}\right]^{\top}, p_{i}, h_{i}, b_{i}$ are the unit energy signature sequence, transmit power, channel gain and information symbol, respectively, of user $i$, and $\mathbf{n}$ is a zero-mean Gaussian random vector with covariance $\sigma^{2} \mathbf{I}_{N}$. The information symbol $b_{i}$ is assumed to have unit energy, i.e., $E\left[b_{i}^{2}\right]=1$. We assume that the receiver and all of the transmitters have perfect knowledge of the channel states of all users represented as a vector $\mathbf{h}=$ $\left[h_{1}, \cdots, h_{K}\right]^{\top}$. We further assume that although the fading is slow enough to ensure constant channel gain in a symbol interval, it is fast enough so that within the transmission time of a block of symbols the long term ergodic properties of the fading process can be observed [13].

For a given set of signature sequences and a fixed set of channel gains, $\mathbf{h}$, the sum capacity $C_{\text {sum }}(\mathbf{h}, \overline{\mathbf{p}}, \mathbf{S})$ is [9],

$$
C_{\text {sum }}(\mathbf{h}, \overline{\mathbf{p}}, \mathbf{S})=\frac{1}{2} \log \left[\operatorname{det}\left(\mathbf{I}_{N}+\sigma^{-2} \sum_{i=1}^{K} h_{i} \bar{p}_{i} \mathbf{s}_{i} \mathbf{s}_{i}^{\top}\right)\right]
$$

where $\bar{p}_{i}$ is the average power of user $i, \overline{\mathbf{p}}=\left[\bar{p}_{1}, \cdots, \bar{p}_{K}\right]$, and $\mathbf{S}=\left[\mathbf{s}_{1}, \cdots, \mathbf{s}_{K}\right]$. To maximize the above capacity for that particular $\mathbf{h}$, one can choose the signature sequences of the users for a given set of power constraints. An equivalent problem is solved in [6], in the no-fading case, i.e., $h_{i}=1$, for all $i$.

In the presence of fading, if the channel state is modelled as a random vector, the quantity $C_{\text {sum }}(\mathbf{h}, \overline{\mathbf{p}}, \mathbf{S})$ is random as well, and the ergodic sum capacity is found as the expected value of $C_{\text {sum }}(\mathbf{h}, \overline{\mathbf{p}}, \mathbf{S})$. Instead of keeping the transmit power of user $i$ fixed to $\bar{p}_{i}$ as in (2), we can choose the transmit powers of the users $p_{i}(\mathbf{h}), i=1, \cdots, K$, as a function of the channel state with the aim of maximizing the ergodic sum capacity of the system subject to average transmit power constraints for all users. Similarly, we can choose the signature sequences $\mathbf{S}$ to be a function of the channel state as well; let us denote it by $\mathbf{S}(\mathbf{h})$ to show the dependence on the channel state. Therefore our problem is to solve for the jointly optimum transmit powers and signature sequences as a function of the channel state in order to maximize the ergodic sum capacity of the system in the presence of fading. The problem can be stated as,

$$
\begin{gathered}
\max _{\mathbf{p}(\mathbf{h}), \mathbf{S}(\mathbf{h})} \int \log \left[\operatorname{det}\left(\mathbf{I}_{N}+\sum_{i=1}^{K} \frac{h_{i} p_{i}(\mathbf{h})}{\sigma^{2}} \mathbf{s}_{i}(\mathbf{h}) \mathbf{s}_{i}(\mathbf{h})^{\top}\right)\right] f(\mathbf{h}) d \mathbf{h} \\
\text { s.t. } \int p_{i}(\mathbf{h}) f(\mathbf{h}) d \mathbf{h}=\bar{p}_{i}, \quad p_{i}(\mathbf{h}) \geq 0
\end{gathered}
$$

where $f(\mathbf{h})$ denotes the probability density function of the channel state vector.

\section{Joint Signature Sequence And Power Allocation}

In order to jointly optimize the powers and signature sequences, we first fix power distributions of all users over all fading states. Then, the corresponding optimal signature sequence set at every channel state will consist of a combination of orthogonal and GWBE sequences [6]. This is due to the fact that, the signature sequences at a fading state $\mathbf{h}$ can be chosen independently of the signature sequences at any other state, since once the powers are fixed, there are no constraints relating $\mathbf{S}(\mathbf{h})$ to $\mathbf{S}(\overline{\mathbf{h}})$ for $\mathbf{h} \neq \overline{\mathbf{h}}$. That is, we can freely choose a sequence set at a given state $\mathbf{h}$ without changing the contribution to the sum capacity of another state $\overline{\mathbf{h}}$; this clearly is not true for the power allocation, since once we allocate a power level for a given state $\mathbf{h}$, we have less power left to allocate to other states, and overall capacity is affected. Since the optimum signature sequences at each channel state depend only on powers $\mathbf{p}(\mathbf{h})$ and the channel 
state $\mathbf{h}$, we can express the capacity at each channel state only as a function of the powers, and optimize the ergodic capacity in terms of the power allocation. Let us define the signature sequence optimized sum capacity at channel state $\mathbf{h}$ for a given power control policy $\mathbf{p}(\mathbf{h})$ by

$$
C_{\text {opt }}(\mathbf{h}, \mathbf{p}(\mathbf{h})) \triangleq \max _{\mathbf{S}(\mathbf{h})} C_{\text {sum }}(\mathbf{h}, \mathbf{p}(\mathbf{h}), \mathbf{S}(\mathbf{h}))
$$

where $C_{\text {sum }}(\mathbf{h}, \mathbf{p}(\mathbf{h}), \mathbf{S}(\mathbf{h}))$ is the argument of the expectation in the objective function of (3), i.e., it is the function in (2) where $\overline{\mathbf{p}}$ is replaced by $\mathbf{p}(\mathbf{h})$ and $\mathbf{S}$ is replaced by $\mathbf{S}(\mathbf{h})$. For a fixed $\mathbf{h}$, it can be shown using majorization theory that $C_{\text {opt }}(\mathbf{h}, \mathbf{p}(\mathbf{h}))$ is a concave function of the power vector at channel state $\mathbf{h}, \mathbf{p}(\mathbf{h})$ [14, Proposition 2.2]. Then, the problem in (3) can be written only in terms of the powers as

$$
\begin{aligned}
& \max _{\mathbf{p}(\mathbf{h})} \int C_{\mathrm{opt}}(\mathbf{h}, \mathbf{p}(\mathbf{h})) f(\mathbf{h}) d \mathbf{h} \\
& \text { s.t. } \int p_{i}(\mathbf{h}) f(\mathbf{h}) d \mathbf{h}=\bar{p}_{i}, \quad p_{i}(\mathbf{h}) \geq 0
\end{aligned}
$$

First consider the case when $K \leq N$. For any fixed channel state, the optimal choice of signature sequences for a given power control policy $\mathbf{p}(\mathbf{h})$ is an orthogonal set [5], [6]. Noting that the received power levels are $p_{i}(\mathbf{h}) h_{i}$, solving (5) is equivalent to solving $K$ independent Goldsmith-Varaiya problems [2] (see also [1]), the solution to which is a single user waterfilling for each user. The optimal solution $\mathbf{p}^{*}(\mathbf{h})$ is the unique solution satisfying the Karush-Kuhn-Tucker (KKT) conditions, and is given by,

$$
p_{i}^{*}(\mathbf{h})=\left(\frac{1}{\lambda_{i}}-\frac{\sigma^{2}}{h_{i}}\right)^{+}, \quad i=1, \cdots, K
$$

where $\lambda_{i}$ is solved by plugging (6) into (5).

One remarkable observation is that in obtaining $C_{\text {opt }}(\mathbf{h}, \mathbf{p}(\mathbf{h}))$, it is possible to adopt a channel non-adaptive signature sequence allocation policy, i.e., each user can be assigned a designated signature sequence, which it can use at all channel states, as long as the signature sequences in this set are orthogonal. A channel adaptive scheme will also perform equally well as long as the signature sequences we choose at each $\mathbf{h}$ are from an orthogonal set.

When $K>N$, it has been shown in [6], for a non-fading channel, that given the power constraints of all users, one can group the users into two sets $L$ and $\bar{L}$, of oversized and nonoversized users, respectively. Users $i \in L$ are assigned orthogonal sequences, and users $i \in \bar{L}$ are assigned GWBE sequences. For a channel with fading, at a certain channel state $\mathbf{h}$, and for a certain arbitrary power distribution of users which assigns powers $p_{1}, \cdots, p_{K}$ to channel state $\mathbf{h}$, let us define the matrix $\mathbf{D} \triangleq$ $\operatorname{diag}\left(p_{1} h_{1}, \cdots, p_{K} h_{K}\right)$, and define $\mu_{i}$ to be the eigenvalues of the matrix $\mathbf{S D S}^{\top}$. Then the signature sequences that maximize the sum capacity for any fixed h satisfy [15],

$$
\mathbf{S D S}^{\top} \mathbf{s}_{i}=\mu_{i} \mathbf{s}_{i}, \quad i=1, \cdots, K
$$

clearly with repetitions of some of the $\mu_{i} \mathrm{~s}$ (since there are only $N$ eigenvalues of $\mathbf{S D S}^{\top}$ ), where the optimal $\mu_{i}$ s are given by [6],

$$
\mu_{i}(\mathbf{h})= \begin{cases}\frac{\sum_{j \in \bar{L}} p_{j} h_{j}}{N-|L|}, & i \in \bar{L} \\ p_{i} h_{i}, & i \in L\end{cases}
$$

In the fading case with channel adaptive powers, as suggested by the results in [1]-[3], it is likely that some users will have powers equal to zero at some channel states, and they will not contribute to $C_{\text {sum }}$ at those channel states. Although the concept of oversized users is defined for users with nonzero average power constraints, since users which are allocated zero power at state $\mathbf{h}$ will not contribute to the sum capacity, we can add them to the set of non-oversized users at channel state $\mathbf{h}, \bar{L}(\mathbf{h})$, and we can assume that we assign arbitrary sequences for those users without changing the solution. Note however that, while finding the set of oversized users, we will disregard the users with zero power. Using the optimum eigenvalue assignment in (8) at each state, the objective function of the problem (5) can be expressed in the alternative form,

$$
\begin{aligned}
\int & {\left[\sum_{i \in L(\mathbf{h})} \log \left(1+\frac{p_{i}(\mathbf{h}) h_{i}}{\sigma^{2}}\right)\right.} \\
& \left.+(N-|L(\mathbf{h})|) \log \left(1+\frac{\sum_{i \in \bar{L}(\mathbf{h})} p_{i}(\mathbf{h}) h_{i}}{\sigma^{2}(N-|L(\mathbf{h})|}\right)\right] f(\mathbf{h}) d \mathbf{h}
\end{aligned}
$$

For a given channel state $\mathbf{h}$, let the set of users that will transmit with non-zero powers be $\bar{K}(\mathbf{h})$. Then the number of users in $\bar{K}(\mathbf{h})$ cannot exceed $N$, as stated by the following theorem.

Theorem 1: Let $\bar{K}(\mathbf{h})$ be a subset of $\{1, \cdots, K\}$, such that $\forall i \in \bar{K}(\mathbf{h}), p_{i}^{*}(\mathbf{h})>0$, where $\mathbf{p}^{*}(\mathbf{h})$ is the maximizer of (9). Then, $|\bar{K}(\mathbf{h})| \leq N$, almost surely.

Proof: By concavity of $C_{\text {opt }}(\mathbf{h}, \mathbf{p}(\mathbf{h}))$, it is clear that the function in (9) is concave, and the maximization in (5) is over an affine set of constraints. Therefore, a power vector $\mathbf{p}^{*}(\mathbf{h})$ achieves the global optimum of the maximization problem if and only if it satisfies the KKT conditions. Then, writing the KKT conditions for the objective function in (9), it is easy to show that

$$
\frac{h_{i}}{\mu_{i}(\mathbf{h})+\sigma^{2}} \leq \lambda_{i}, \quad \forall \mathbf{h}
$$

where $\mu_{i}(\mathbf{h})$ is given by (8), and equality holds if $p_{i}(\mathbf{h})>0$. Now, let us assume that the number of non-zero components in $\mathbf{p}^{*}(\mathbf{h})$ is $|\bar{K}(\mathbf{h})|>N$, for a given $\mathbf{h}$. Then, some users must share some of the available dimensions, i.e., not all users can be made orthogonal to each other. In fact, we can find at most $N-1$ sequences that are orthogonal to all other sequences in the system, or equivalently, at least $|\bar{K}(\mathbf{h})|-N+1$ users will have the same $\mu_{i}=\sum_{j \in \bar{L}(\mathbf{h})} h_{j} p_{j} /(N-|L(\mathbf{h})|)$. Then, substituting this into (10), we get $h_{i} / \lambda_{i}=h_{j} / \lambda_{j}$ for $i \neq j, i, j \in \bar{K}(\mathbf{h})$ for at least $|\bar{K}(\mathbf{h})|-N+1$ users. Note that as the channel fading is assumed to be a continuous random variable, this event has zero probability, and at most one user with GWBE sequences (one with highest $h_{i} / \lambda_{i}$ ratio, as in [3]) may transmit, with probability 1. But this contradicts the assumption that $|\bar{K}(\mathbf{h})|>N$, which establishes our main result, i.e., $|\bar{K}(\mathbf{h})| \leq N$ almost surely. 
An important implication of this result is, since the optimal power allocation dictates that at most $N$ users transmit with positive powers, orthogonal sequences should be assigned to those users that are transmitting with positive powers. That is, although we allowed for allocating GWBE sequences to some of the users, the solution implies that there is at most one such user, and the problem reduces to the orthogonal case. The optimal power allocation is again single user waterfilling, similar to the solution given in (6), i.e.,

$$
p_{i}^{*}(\mathbf{h})= \begin{cases}\left(\frac{1}{\lambda_{i}}-\frac{\sigma^{2}}{h_{i}}\right), & i \in \bar{K}(\mathbf{h}) \\ 0, & \text { otherwise }\end{cases}
$$

Here, one needs to be careful about the transmit regions. Unlike the case where the actual number of users $K \leq N$, the users in the set $\bar{K}(\mathbf{h})$ change with $\mathbf{h}$, thus a channel adaptive allocation of the orthogonal sequences is necessary. Our convention is, we assign a sequence from an orthogonal set to a user, wherever its power is positive.

To specify the optimal power allocation completely, let us define $\gamma_{i}=h_{i} / \lambda_{i}$. Then, the probability that $\gamma_{i}=\gamma_{j}$, for $i \neq j$ is zero. Therefore, we can always find a unique order statistics $\left\{\gamma_{[i]}\right\}_{i=1}^{K}$ such that $\gamma_{[1]}>\cdots>\gamma_{[K]}$, for each given $\mathbf{h}$. Let us now place $\sigma^{2}$ in that ordering, assuming that at least one of the $\gamma_{[i]} \mathrm{s}$ is larger than $\sigma^{2}$. Define $\gamma_{[K+1]}=0$. Then, for some $n \in\{1, \cdots, K\}$, let

$$
\gamma_{[1]} \geq \cdots \geq \gamma_{[n]}>\sigma^{2} \geq \gamma_{[n+1]} \geq \cdots \geq \gamma_{[K+1]}
$$

where the equalities are included for the sake of consistency of the indices, and do not affect the solution (note the strict inequality just before $\sigma^{2}$ ).

First, let $n \leq N$. Then, we see that (11) gives positive powers for all $n$ users, and thus all $n$ users with highest $\gamma_{i}$ s will transmit with the non-zero powers given in (11). When $n>N$, there are more than $N$ users satisfying the positivity constraints $\gamma_{i}>\sigma^{2}$. However, we know from our derivation that only the user with the highest $\gamma_{i}$ from the set we intend to assign GWBE sequences may transmit. Therefore, a total of $N$ users with the highest $\gamma_{i} \mathrm{~s}$ transmit at this channel state.

Finally, we can summarize the jointly optimal power and signature sequence allocation policy as,

$$
\begin{aligned}
& p_{i}^{*}(\mathbf{h})= \begin{cases}\left(\frac{1}{\lambda_{i}}-\frac{\sigma^{2}}{h_{i}}\right), & \text { iff } i \in \Omega \\
0, & \text { otherwise }\end{cases} \\
& \mathbf{s}_{i}^{*}(\mathbf{h})^{\top} \mathbf{s}_{j}^{*}(\mathbf{h})=0, \quad i \neq j, \quad \forall i, j \in \Omega \\
& \Omega=\left\{i: \gamma_{[i]}>\sigma^{2}, \quad i \leq \min \{K, N\}\right\}
\end{aligned}
$$

\section{ITERATIVE Power AND Sequence Optimization}

We found in the previous section that the optimal power control strategy is a waterfilling over some favorable fading states for each user. In [1], it was shown that a one-user-at-a-time waterfilling algorithm converges to an optimum simultaneous waterfilling solution, that maximizes the ergodic sum capacity for a fixed set of signature sequences. This result motivates us to develop a similar algorithm for the joint signature sequence and power optimization problem.
In [1], we have shown that for fixed signature sequences $\mathbf{S}$, the optimal single-user update that maximizes the sum capacity as a function of $p_{k}(\mathbf{h})$ is given by,

$$
p_{k}(\mathbf{h}, \mathbf{S})=\left(\frac{1}{\lambda_{k}}-\frac{1}{h_{k} \mathbf{s}_{k}^{\top} \mathbf{A}_{k}^{-1} \mathbf{s}_{k}}\right)^{+}
$$

where the interference covariance matrix $\mathbf{A}_{k}$ is defined as,

$$
\begin{aligned}
\mathbf{A}_{k} & =\sigma^{2} \mathbf{I}_{N}+\sum_{i \neq k} h_{i} p_{i}(\mathbf{h}) \mathbf{s}_{i} \mathbf{s}_{i}^{\top} \\
& =\sigma^{2} \mathbf{I}_{N}+\mathbf{S D S}^{\top}-h_{k} p_{k}(\mathbf{h}) \mathbf{s}_{k} \mathbf{s}_{k}^{\top}
\end{aligned}
$$

We can find and fix the optimal signature sequences at each state for a given power allocation using results of [6]. Then, plugging these sequences in (15), and noting that the signature sequences that maximize the sum capacity for a fixed set of power constraints satisfy (7), we get

$$
\mathbf{A}_{k} \mathbf{s}_{k}^{*}=\left(\sigma^{2}+\mu_{k}-h_{k} p_{k}\right) \mathbf{s}_{k}^{*}
$$

where $\mu_{k}$ are given by (8). Therefore,

$$
\mathbf{s}_{k}^{* \top} \mathbf{A}_{k}^{-1} \mathbf{s}_{k}^{*}=\frac{1}{\sigma^{2}+\mu_{k}-h_{k} p_{k}}
$$

This shows that, we can represent the base level for the waterfilling in (14) as a function of the power levels in the previous iteration. Substituting this in (14), we get the optimal power allocation at the $n+1$ st step, $p_{k}^{n+1}(\mathbf{h})$ for user $k$, with optimal sequences and fixed powers $\left\{p_{i}(\mathbf{h})\right\}_{i \neq k}$ from the previous iteration

$$
p_{k}^{n+1}(\mathbf{h})=\left(\frac{1}{\lambda_{k}}-\frac{\sigma^{2}+\mu_{k}^{n}(\mathbf{h})-h_{k} p_{k}^{n}(\mathbf{h})}{h_{k}}\right)^{+}
$$

where we use $\left\{p_{1}^{n+1}(\mathbf{h}), \cdots, p_{k-1}^{n+1}(\mathbf{h}), p_{k}^{n}(\mathbf{h}), \cdots, p_{K}^{n}(\mathbf{h})\right\}$ to compute $\mu_{k}^{n}(\mathbf{h})$. Combining this with (8) gives us the power update at each step. It is easy to observe that, once the eigenvalues $\mu_{k}^{n}(\mathbf{h})$ are determined using the power levels from the previous iteration, we can use (18) to solve for $k$ th user's power by waterfilling.

The proposed algorithm may be interpreted in two ways. First, it may be seen as an iteration from a set of powers to another set of powers as given by (18). Therefore, one may run this algorithm starting with an arbitrary power distribution, to obtain the capacity maximizing power distribution when the algorithm converges. The signature sequences may then be assigned to the users after the algorithm converges: at each channel state, the users that have non-zero powers (there will be at most $N$ such users) are assigned signature sequences from an orthogonal set. Second, the algorithm may be seen as an iteration from powers to signature sequences, and then back to powers again. Specifically, for a given set of powers, the optimal sequences may be found using (7) and (8), i.e., as in [6]; corresponding to these sequences, base levels for the waterfilling in (14) can be computed using (16) and (17), and new powers may be found using (14) as in [1].

We will now show that (18) and equivalently the sequential signature sequence and power update algorithm indeed converges to the global optimum of the sum capacity function. To see this, first observe that for fixed signature sequences, the update (14) 


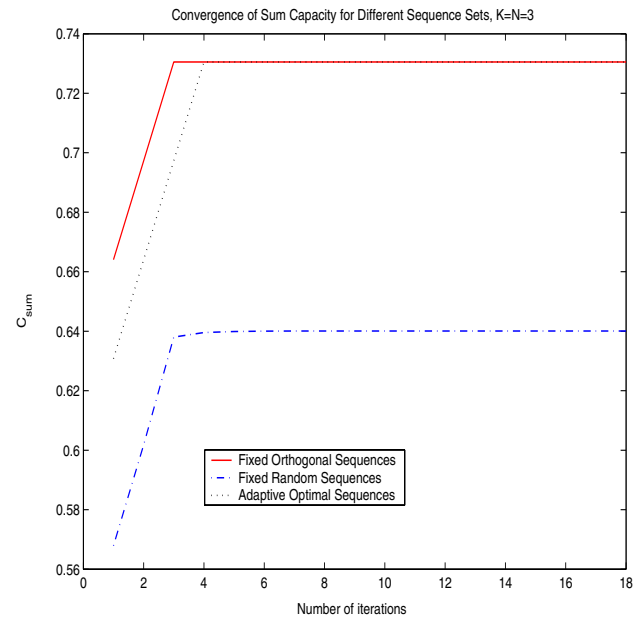

Fig. 1. Convergence of sum capacity for $\mathrm{K}=\mathrm{N}=3$.

is the best one-user-at-a-time power update and is guaranteed to give a non-decreasing sequence of sum capacity values [1]. Similarly, for fixed powers, the signature sequence update will increase (or keep constant) the value of the sum capacity. The sum capacity is upper bounded, therefore it is guaranteed that the sequence of non-decreasing sum capacity values obtained through these iterations have a limit. Moreover, the algorithm terminates if and only if the update (18) yields a fixed point $\mathbf{p}(\mathbf{h})$. Since the fixed point is characterized by $\mathbf{p}^{n+1}=\mathbf{p}^{n}$, it is easy to see that the fixed point of the update (18) actually satisfies the KKT conditions for our original problem. Since the convergence point $\mathbf{p}(\mathbf{h})$ satisfies the KKT conditions, it achieves the global optimum of the sum capacity, proving the convergence of the sequential algorithm.

Next, we demonstrate the performance and results of the above algorithm. In all of our simulations, we pick $\sigma^{2}=1$, the average power of each user to be 1 , the initial power distribution uniformly, and the probability distribution of the channel to be uniform. Firstly, we simulate a system where the number of users is equal to the processing gain: $K=N=3$. In this case, by our arguments in Section III, we expect the optimal signature sequences to be three orthogonal sequences. Figure 1 shows the convergence of our algorithm, together with the convergence of the iterative waterfilling algorithm in [1] for fixed sequences. When we optimize the powers and signature sequences jointly, we see that the sum capacity achieved is identical to that of a system with fixed orthogonal sequences, meaning channel adaptive and non-adaptive sequence selections give us the same capacity value. Our algorithm in this case converges to the optimum in one round of iterations (one iteration for each user). The capacity achieved by a randomly generated signature sequence matrix $\mathbf{S}$ containing unit-norm sequences is also given for comparison.

The convergence plots for a more interesting case where $K=4, N=3$ are given in Figure 2. Here, we again compare the capacity achieved by our algorithm to some fixed randomly generated sequences, and we see that we get a higher capacity. We also compare our result to a fixed set of WBE sequences,

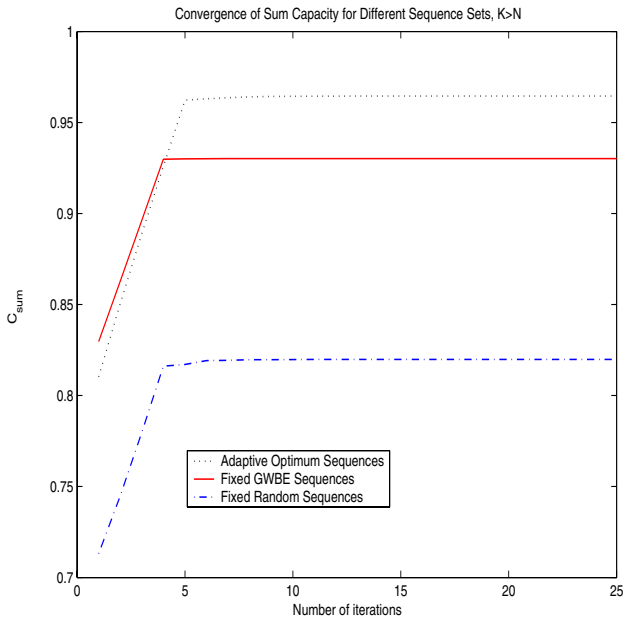

Fig. 2. Convergence of sum capacity for $K=4, N=3$.

which are the optimum sequences for a fixed channel state and equal average received powers. The iterative waterfilling with sequence optimization again achieves a better sum capacity. Also remarkably, the transmit strategy is such that at most 3 of the 4 users transmit together (on a region with non-zero probability, after eliminating the states where the channel states of any two users are equal), and they are allocated orthogonal sequences.

\section{REFERENCES}

[1] O. Kaya and S. Ulukus. Optimum power control for fading CDMA with deterministic sequences. In Allerton Conference, Monticello, IL, Oct. 2002.

[2] A. J. Goldsmith and P. P. Varaiya. Capacity of fading channels with channel side information. IEEE Trans. Info. The., 43(6):1986-1992, November 1997.

[3] R. Knopp and P. A. Humblet. Information capacity and power control in single-cell multiuser communications. In IEEE ICC, June 1995.

[4] R. D. Yates. A framework for uplink power control in cellular radio systems. IEEE JSAC, 13(7):1341-1347, September 1995.

[5] M. Rupf and J.L. Massey. Optimum sequence multisets for synchronous code-division multiple-access channels. IEEE Trans. Info. The., 40:12611266, July 1994.

[6] P. Viswanath and V. Anantharam Optimal sequences and sum capacity of Synchronous CDMA systems. IEEE Trans. Info. The., 45(6):1984-1991, November 1999.

[7] P. Viswanath, V. Anantharam and D. Tse. Optimal Sequences, Power Control and Capacity of Synchronous CDMA Systems with Linear MMSE Multiuser Receivers. IEEE Trans. Info. The., 45(6):1968-1983, Sept. 1999.

[8] T. M. Cover and J. A. Thomas. Elements of Information Theory. Wiley Interscience, 1991.

[9] S. Verdú. Capacity region of Gaussian CDMA channels: The symbolsynchronous case. In Allerton Conference, Monticello, IL, pages 1025-1034, October 1986.

[10] P. Viswanath, D. Tse, and V. Anantharam. Asymptotically optimal waterfilling in vector multiple access channels. IEEE Trans. Info. The., 12(4):241267, January 2001.

[11] L.R. Welch Lower bounds on the maximum cross-correlation of signals. IEEE Trans. Info. The., vol. 20, pp. 397-399, 1974.

[12] S. Verdú. Multiuser Detection. Cambridge University Press, 1998.

[13] E. Biglieri, J. Proakis, and S. Shamai (Shitz). Fading channels: Informationtheoretic and communications aspects. IEEE Trans. Info. The., 44(6):26192692, October 1998.

[14] P. Viswanath and V. Anantharam Optimal Sequences for CDMA Under Colored Noise: A Schur-Saddle Function Property. IEEE Trans. Info. The. 45(6):1969-1983, June 1999.

[15] S. Ulukus and R. D. Yates. Iterative Construction of Optimum Signature Sequence Sets in Synchronous CDMA Systems, IEEE Trans. on Info. The., 47(5):1989-1998, July 2001. 